\title{
A New Integral Representation of the Bessel Coefficients
}

\section{By P. Razelos}

The modified Bessel coefficients $I_{n}$ are defined by the series [1]

$$
I_{n}(t)=\sum_{q=0}^{\infty} \frac{\left(\frac{t}{2}\right)^{2 q+n}}{q !(q+n) !}
$$

with the integral representation [1]

$$
I_{n}(t)=\frac{1}{\pi} \int_{0}^{\pi} e^{t \cos x} \cos n x d x .
$$

An integral representation of the coefficients $I_{n}(t)$ is presented here where the path of integration is extended to infinity.

TheOREM: The integral

$$
A_{n}(t)=\frac{2}{\pi} \int_{0}^{\infty} e^{t \cos x} \cos n x \frac{\sin \epsilon x}{x} d x
$$

is the modified coefficient $I_{n}(t)$ for any $0<\epsilon<1$. There are many ways by which the theorem can be proved, but we give here the following proof which consists of a straightforward evaluation of the integral $A_{n}$. The function $e^{t \cos x}$ is expanded in a power series (which is absolutely convergent for all $t$ ) and we then integrate term by term.

$$
e^{t \cos x}=\sum_{r=0}^{\infty} \frac{(t \cos x)^{r}}{r !}
$$

Let us define

$$
Q_{n}^{r}=\frac{2}{\pi} \int_{0}^{\infty} \cos ^{r} x \cos n x \frac{\sin \epsilon x}{x} d x
$$

Then

$$
A_{n}(t)=\sum_{r=0}^{\infty} \frac{Q_{n}{ }^{r}}{r !} t^{r}
$$

Introducing the expansion

$$
\cos ^{r} x=\frac{1}{2^{r-1}} \sum_{k=0}^{(r / 2)-\delta}\left(\begin{array}{l}
r \\
k
\end{array}\right) \cos (r-2 k) x
$$

(where $\delta=1$ or $\frac{1}{2}$ for $r$ even or odd, respectively) into (4), we obtain

$$
Q_{n}^{r}=\frac{1}{2^{r}} \sum_{k=0}^{(r / 2)-\delta}\left(\begin{array}{l}
r \\
k
\end{array}\right)[g(r-2 k+n)+g(r-2 k-n)],
$$

Received March 31, 1964. Revised July 6, 1964. 
where

$$
g(y)=\frac{2}{\pi} \int_{0}^{\infty} \cos y x \frac{\sin \epsilon x}{x} d x=0,1, \frac{1}{2}
$$

for $|y / \epsilon|$ greater than, less than, or equal to one, respectively [2]. Therefore, the only term which is nonzero in ( 7$)$ is the term $g(0)$, if it exists.

Then

$$
Q_{n}{ }^{r}=\frac{1}{2^{r}} \frac{r !}{\left(\frac{r-n}{2}\right) !\left(\frac{r+n}{2}\right) !}, \quad r \geqq n \text { and } r, n \text { both even or odd, }
$$

(10) $Q_{n}{ }^{r}=0$ for $r<n$ or $r, n$ one even, one odd.

We can now write

$$
\begin{aligned}
& r-n=2 q, \\
& r+n=2(q+n) .
\end{aligned}
$$

Thus,

$$
Q_{n}^{r}=\frac{r !}{2^{r} q !(q+n) !} .
$$

Substituting (12) into (5), we obtain

$$
A_{n}(t)=\sum_{q=0}^{\infty} \frac{\left(\frac{t}{2}\right)^{2 q+n}}{q !(q+n) !}=I_{n} . \quad \text { Q.E.D. }
$$

A similar expression can be readily obtained for the coefficients $J_{n}(t)$. The value of $\epsilon=\frac{1}{2}$ gives the following interesting result. Let us define

$$
B_{n}(t)=\frac{2}{\pi} \int_{n-(1 / 2)}^{n+(1 / 2)} \int_{0}^{\infty} e^{t \cos x} \cos \nu x \frac{\sin (x / 2)}{x} d \nu d x .
$$

It can be easily shown that $B_{n}(t)=I_{n}(t)$. Consider now the integral

$$
\begin{aligned}
B(t) & \doteq \frac{2}{\pi} \int_{-\infty}^{\infty} I_{\nu}\left(\frac{1}{2}, t\right) d \nu \\
& =\frac{2}{\pi} \int_{0}^{\infty} d \nu \int_{0}^{\infty} 2 e^{t \cos x} \cos (\nu x) \frac{\sin (x / 2)}{x} d x \\
& =\lim _{y \rightarrow 0} \frac{2}{\pi} \int_{0}^{\infty} d \nu \int_{0}^{\infty} 2 e^{t \cos x} \cos (\nu x) \cos (\nu y) \frac{\sin (x / 2)}{x} d x \\
& =\left.2 e^{t \cos y} \frac{\sin y / 2}{y}\right|_{y=0}=e^{t}
\end{aligned}
$$

by Fourier's theorem. Clearly $B(t)=\sum_{-\infty}^{\infty} B_{n}(t)$. 
Acknowledgment. The author wishes to thank Professor H. G. Elrod, Jr. for suggesting the problem.

Columbia University

New York, New York

1. G. N. Watson, A Treatise on the Theory of Bessel Functions, 2nd ed., Cambridge Univ. Press, New York, 1944. MR 6, 64.

2. R. S. Burington, Handbook of Mathematical Tables and Formulas, 3rd ed., Handbook Publishers Inc., Sandusky, Ohio, 1949. MR 22 \#2514.

\section{Footnote to the Evaluation of Certain Complex Elliptic Integrals}

\section{By C. D. Sutherland}

The formulas for evaluating the elliptic integral of the third kind wi $h$ a complex parameter as given by Byrd and Friedman [1] have been corrected and simplified Iby Lang and Stevens [2]. There is, however, a further correction necessary in these latter results.

The integral to be evaluated is

$$
I=\left(a_{1}+i b_{1}\right) \int_{0}^{\phi} \frac{d \theta}{\left(1-\alpha^{2} \sin ^{2} \theta\right) \Delta},
$$

where $\alpha^{2}$ is complex and $\Delta=\sqrt{ }\left(1-k^{2} \sin ^{2} \theta\right)$. In the formulas for evaluating $I$ there appears the quantity

$$
\tau_{2}=\int_{0}^{p_{2}} \frac{m_{2} d x}{1+h_{2} x^{2}}=\frac{m_{2}}{\sqrt{ } h_{2}} \tan ^{-1}\left(p_{2} \sqrt{ } h_{2}\right)
$$

where

$$
p_{2}=\frac{\sin \phi \cos \phi}{\left(1+m_{2} \sin ^{2} \phi\right) \Delta} .
$$

We will consider the case where $m_{2} \leqq-1$. If this occurs we see that as $\phi$ goes to $\pi / 2$, either $p_{2} \rightarrow \infty\left(m_{2}=-1\right)$ and $\left[\tan ^{-1}\left(p_{2} \sqrt{ } h_{2}\right)\right] \rightarrow \pi / 2$, or $p_{2} \rightarrow 0$ through negative values $\left(m_{2}<-1\right)$ and $\left[\tan ^{-1}\left(p_{2} \sqrt{ } h_{2}\right)\right] \rightarrow \pi$ (and not to zero). To avoid overlooking this possibility the proper representation for $\tau_{2}$ is

$$
\begin{aligned}
\tau_{2} & =\frac{-1}{\sqrt{ } h_{2}} \cos ^{-1}\left(\frac{\Delta \cos \phi}{\sqrt{ }\left(h_{2} \sin ^{2} \phi+\Delta^{2} \cos ^{2} \phi\right)}\right) \text { for } m_{2}=-1 \\
\tau_{2} & =\frac{m_{2}}{\sqrt{ } h_{2}} \cos ^{-1}\left(\frac{\Delta\left(1+m_{2} \sin ^{2} \phi\right)}{\sqrt{ }\left(h_{2} \sin ^{2} \phi \cos ^{2} \phi+\Delta^{2}\left(1+m_{2} \sin ^{2} \phi\right)^{2}\right)}\right) \text { for } m_{2} \neq-1 .
\end{aligned}
$$

It is to be noted, in particular, that the formulas for the real and imaginary parts of the complete integral should contain a term involving $\tau_{2}$ whenever $m_{2} \leqq-1$.

Received March 16, 1964. Work performed under the auspices of the U. S. Atomic Energy Commission. 